\title{
Remote Expert System of Poultry Farming Based on Internet of Things
}

\author{
Xiangli XIN \\ Department of information technology, Beijing College of Politics and Law, Huangyi Road, DaXing \\ District, Beijing, China \\ email:xinx174@163.com
}

\section{Keywords: Poultry farming; Technology of internet of things; Expert system}

\begin{abstract}
In order to make it easy for poultry farming staff to seek help and diagnosis for poultry diseases, especially emergent and disastrous situations, enhance the effectiveness, rapidness and simplicity of the service, and avoid cross infection caused by experts walking from a farming area to another farming area, the technology of internet of things is applied into the remote diagnosis expert system for the poultries to collect the environment information of the farming area such as temperature and humidity, ventilation, light, ammonia gas and on-site video and image, so that the remote expert system can achieve real-time diagnosis, prevention and treatment of the diseases. With the system, poultry farmers can seek real-time help online and experts can give on-site guidance to the farmers in the process of disease prevention and treatment, which will reduce the occurrence of poultry diseases and enhance the earnings of the farmers.
\end{abstract}

\section{Introduction}

With the improvement of people's consumption level and a considerable transfer of labor force from agricultural production, the agriculture has displayed a trend of intensified production and operation, the modern farming industry is expanding and developing rapidly [1]. However, due to the shortage of poultry farming enterprises in the areas such as industrialization level, the technical level of farming staff, technical devices of farming and production process management, the farming equipment is low in technological content, which is still dominant with manpower. The labor intensity and human cost are high while the productivity and intelligentization level are relatively low, as a result of which the farming environment is poor and the output and quality are affected. In order to solve the above problems, we must rely on agricultural technology and promote the transformation and upgrade of poultry farming industry [2]. We are in an urgent need to rely on a new generation of information technologies such as internet of things, achieve automatic, precise, clean and intelligent production and operation of poultry farming, truly reduce the labor cost and enhance the output and quality [3]. Therefore, it's pressing to carry out research and demonstrative application of technologies and products of internet of things for intensified and healthy farming of poultry. The research of farming expert system has begun to be applied in the diagnosis of poultry diseases since the end of 1980s [4]. In 1999, experts of Beijing Youge Technology Development Co., Ltd., the Feed Industry Center under the Ministry of Agriculture and the Institute of Zoology of Chinese Academy of Science jointly developed a new generation of intelligent poultry clinical software, which had a diagnosis accuracy rate of above 95\% [5]. After many years' development, the poultry diseases diagnosis has become one of the most dynamic fields for the agricultural application of expert system, with a good research foundation and application prospect.

The various poultry expert diagnosis systems adopted today has achieved certain success, but there are also certain shortages. For example, all standalone versions are called expert system, but they are not real-time expert diagnosis and are not suitable for today's network environment; some systems display deficiencies and limitations such as incomplete knowledge, simple reasoning method [6], and inappropriate application of knowledge and thus are not extensive in the scope of application. Especially most expert systems receive and process text information. However, the farmers usually cannot accurately describe the disease characteristics, as a result of which the expert system cannot make a correct judgment, which then requires the introduction of multi-media description method and 
a more accurate diagnosis mechanism [7]. The technology of internet of things emerging and developing in recent years has unique advantages in the monitoring and management of poultry farming [8]. Through information sensing equipment such as RFID, sensor, global positioning system and laser scanner, based on the agreed protocol, the internet of things connects any thing with the internet for information exchange and communication, thereby realizing intelligentized identification, positioning, tracking, monitoring and management. It's a network connecting with the physical world with the features such as comprehensive sensing, reliable transmission and intelligent processing, which has realized the connection of any time, any place and any object. It can help achieve an organic combination between the human society and the physical world, let the human manage production and life in a more exquisite and dynamic manner, thereby enhancing the informationization capacity of the whole society. Therefore, the remote expert diagnosis system for poultry diseases based on the technology of internet of things is extremely necessary. The system can effectively help the farming industry save costs and enhance efficiency. The promotion of the technology will become an effective measure to help the farmers to diagnose the poultry diseases and increase income.

\section{System Design}

Overall Architecture. With the collecting modules of each terminal sensor (such as collecting modules of temperature and humidity, light and ammonia gas), the collected information is transmitted via ZigBee protocol to the master controller and constructs a wireless sensing network with the master controller to achieve interconnection and interworking. The master controller transmits the information via the internet to the PC. The farmers transmit the collected farming environment information, disease symptoms description and the videos and images captured by the camera to the server [9]. Receiving the help information, the server obtains diagnosis result via the expert system or the expert online and then feedbacks the result to the farmers. The overall architecture of the system is shown in Fig. 1.

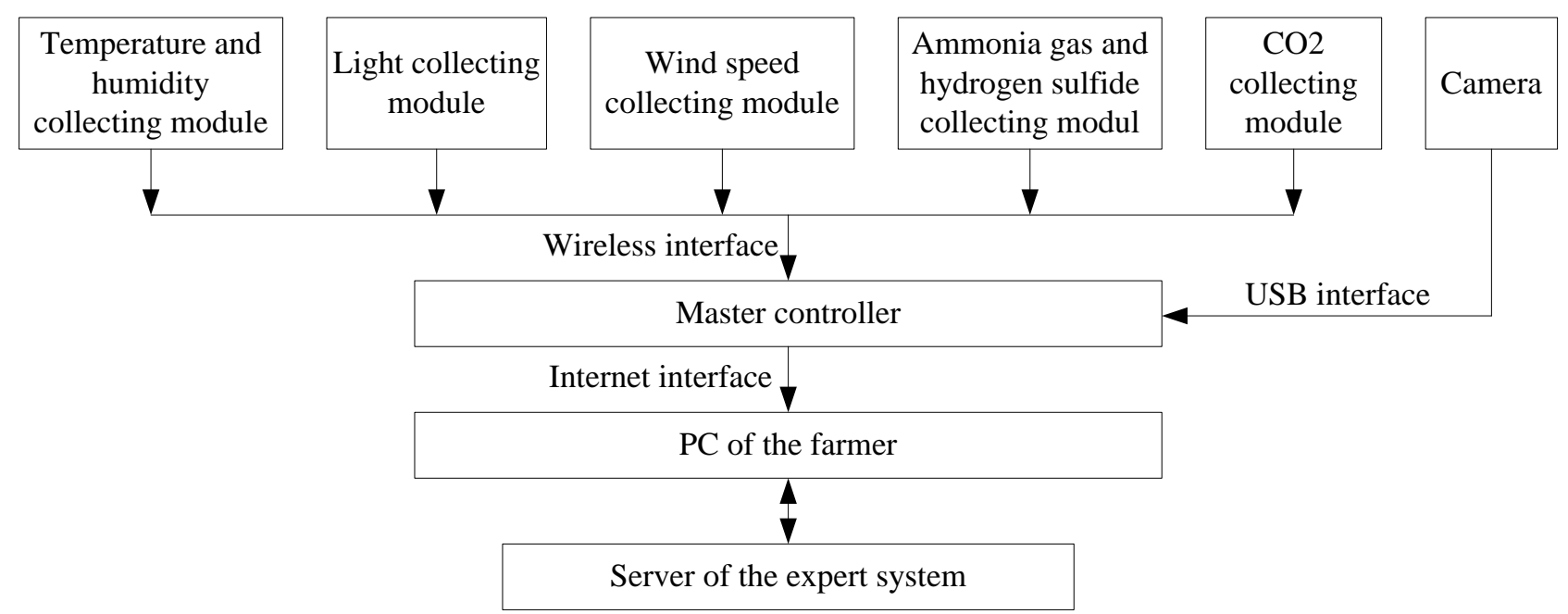

Fig.1. Overall Architecture of the System

Design of sensor nodes. Appropriate sensors are selected based on the parameters of the farming area such as temperature, ventilation, light, ammonia gas, hydrogen sulfide and $\mathrm{CO}_{2}$ to construct collecting nodes of the sensors. For the temperature and humidity sensor, JCJ100S model of JUSCAN Technology wall-mounted temperature and humidity sensor is selected, with a measuring range of temperature $0-50{ }^{\circ} \mathrm{C}$, a precision of $\pm 1{ }^{\circ} \mathrm{C}$, a measuring range of humidity at $25-90 \%$ and a precision of $\pm 2 \%$, and outputting a current signal of 4 -20mA. For the light sensor, JCJ100P light sensor is selected, with a measuring range of 0 -20000Lux, a precision of $\pm 7 \%$ and outputting a current signal of $4-20 \mathrm{~mA}$. For the ammonia and hydrogen sulfide sensor, the BMG800 series intelligent gas transmitter is selected, with a measuring range of ammonia gas at $0-100 \mathrm{ppm}$, a 
precision of $\pm 3 \%$ and a response time of 30 seconds and with a measuring range of hydrogen sulfide at $0-100 \mathrm{ppm}$, a precision of $\pm 3 \%$ and a response time of 45 seconds and outputting a current signal of 4-20mA. For the $\mathrm{CO}_{2}$ sensor, the infrared $\mathrm{CO}_{2}$ sensor of US-based Telaire Company is selected, with a measuring range of $0-2000 \mathrm{ppm}$ and a precision of $\pm 4 \%$ and outputting a current signal of 4-20mA. For the wind speed sensor, the model BS1-EE65-C is selected, with a range of wind speed at $0-20 \mathrm{~m} / \mathrm{s}$, outputting a current signal of $4-20 \mathrm{~mA}$ and a precision reaching $\pm 0.1 \mathrm{~m} / \mathrm{s}$. The collecting nodes of the sensors are responsible for converting environment information into digital signal and transmitting the signal to the master controller through the ZigBee protocol. In terms of the current research and development level, IEEE 802.15.4 is the best scheme for wireless sensor application. Currently there are several companies supplying transceiver chips of this kind. In the design CC2430/CC2431/CC2530 wireless single chips based on 8051 inner core are selected to construct nodes of the sensors.

Building the system of internet of things. The internet of things for poultry farming is generally a fully distributed system applying a kind of acentric nodes, namely, a wireless sensor network. Through the way of random distribution, a considerable number of sensor nodes are densely deployed at the monitoring area. These sensor nodes are integrated with sensors, data processing units, communication modules and energy units. They are connected with each other via wireless channels and self-organized to form a network system. Its aim is to collaboratively perceive, collect and process the information of monitored objects in the area covered by the network and send the information to the observer to help the farmers to timely identify the problem and correctly determine the position of the problem. The research constructs an internet of things through ZigBee protocol with the master controller and sensor nodes of temperature and humidity, ventilation, light, ammonia gas and hydrogen sulfide and $\mathrm{CO}_{2}$. Its structure is shown in Fig. 1. In the construction of sensor nodes, other than the collection of farming environment information, the transceiving and networking of the information of each sensor node is required and the hardware platform of master controller should be developed independently. The master control core of the hardware platform selects ARM11 as the master controller, which can run operating systems such as Linux, WinCE and Android. The peripheral integration includes various interfaces including internet access. The chip CC2530 of ZigBee protocol is selected to achieve communication with each sensor node. Through the Network Stack running on the ARM11 operating system, the wireless sensing network is constructed.

Constructing data collection database, model database and method database. Constructing a complete data transceiving and storage facility, qualitative problems are solved in the form of knowledge reasoning in the expert system. The expert system (as shown in Fig.2) has a regularized form of knowledge and is good at solving the problems under a common situation. The relationship between various farm information and data is stored into the knowledge base to be used as the operating rule for the system under normal conditions and the coping strategy for accidents and provide the expert system with data resource. The expert system is a comprehensive system integrating knowledge base management system, database management system, model base management system and method base management system. The poultry farming and production system, as an open system, shows characteristics of self-organization to a great extent. In the construction of the expert system, C/S structure is adopted, namely, Client/Server model. The computer used by the farmers for receiving and transmitting the data of internet of things is Client computer. Client computers can visit the expert system on the server. Client computers need to process and integrate the collected farm environment information, disease symptoms information and photos and images information and then seek the service of expert system via the server. The principle of "functional distribution" to which it is based has effectively utilized the computing resources of the client computers, which can realize the connection and service of many client computers. The processing mode in which the client computer raises a request and the server provides service fully manifests the high efficiency of the expert system and achieves a huge extension in its service objects. Under a specific environment, the interaction course of the poultry farming system and market environment, at the macroscopic level can be described as the relationship between some order parameters in the synergetics theory. Poultry farming system can be researched as multi-layered 
system in order to establish the corresponding mathematics model .The general multi-layered basic kinetic can be expressed using Liouville equation. The n-dimensional quantum $\mathbf{A}_{j}(\boldsymbol{\Gamma})(j=1 \ldots n)$ meets formula 1 .

$$
\dot{\mathbf{A}}_{j}=i \mathbf{L} \mathbf{A}_{j}
$$

The $\mathrm{L}$ is Liouville operator. $\boldsymbol{\Gamma}$ is phase space point. In general, the $\mathrm{L}$ is a linear operator which is constructed by Hamiltonian system, but the actual biological systems and environment are a closely ralated open system. Besides biological systems are active systems which can not be described as simple potential field. So the L must be nonlinear and contain differential operation. Supposing the basic form of the L operator is formula 2:

$$
\mathbf{L}=\mathbf{L}_{0}-i a \nabla^{2}+i b \mathbf{S}
$$

The $\mathrm{L}_{0}$ is linear hermitian operator, $\mathrm{a}$ and $\mathrm{b}$ is constant, the $\mathrm{S}$ is nonlinear operator whose definition is formula 3 :

$$
\mathbf{S A}_{j}(\vec{x}, \Gamma)=\mathbf{A}_{i}(\vec{x}, \Gamma) \mathbf{A}_{i}^{*}(\vec{x}, \Gamma) \mathbf{A}_{j}(\vec{x}, \Gamma)
$$

Biological systems are highly ordered and complex, it is not possible that understands the global system by solving each sub system, so what we just need to do is study their evolution by finding a few appropriate variables.

These order parameters have summarized the complex interaction mode limiting each sub-system. Since the evolution process of the system is described with a group of differential equations while the properties of the solution to differential equations are determined by a group of characteristic roots. The group of characteristic roots is composed of two parts, namely, the positive discreet system is larger and the negative discreet system is smaller. Since the group of differential equations is non-linear, the characteristic roots will also change with the time.

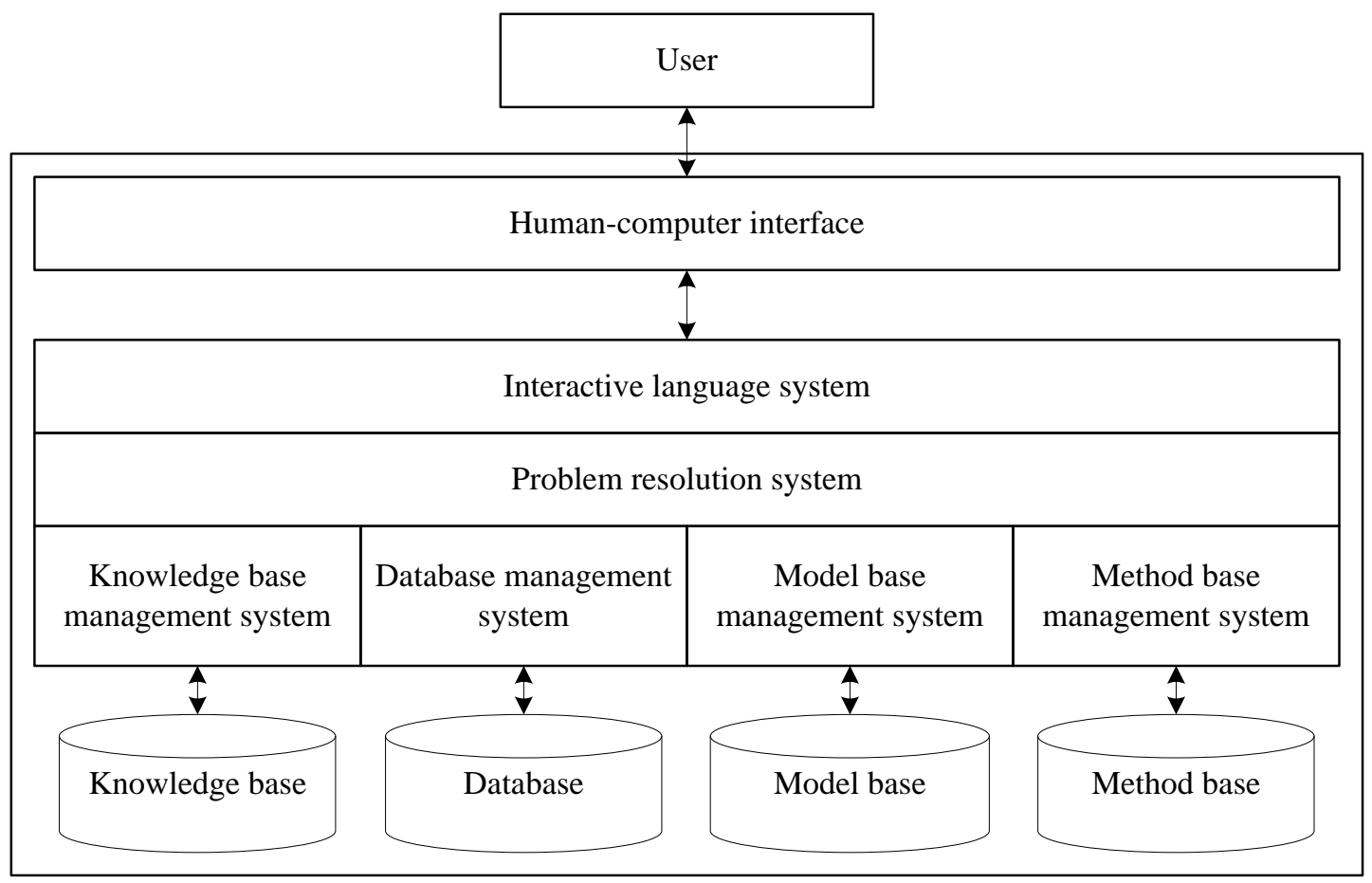

Fig.2. Architecture of the Expert System

In the reasoning process of the remote expert diagnosis system of poultry diseases, firstly it makes a keyword search based on the symptoms information entered or selected by the user. If it matches, the reasoning will be made and then output the results; if the results do not meet the expectation of the user, another computation can be made based on the new information provided by the farmers; if some diseases are encountered that are not included in the information base of poultry diseases, the farmers can apply to the expert online platform to have a real-time communication with the experts 
online. Besides, if some special case is encountered, new knowledge should be added and the knowledge base should be modified.

\section{Conclusions}

A wireless local area network is established to collect the information to the database. Through corresponding sensors, it will capture environment information of the farming area such as temperature, humidity, light, ventilation and concentration of ammonia gas. And through ZigBee wireless communication technology, the collected information is transmitted to the master controller. On this basis, a wireless sensing network is established. A spatial database is developed to access original data and design and develop a model database, including the transformation and determination of data formats, the establishment of a computing model, design and development of method database, accessing different decision schemes (computing models) to compare the decision scheme obtained from the model database with the method database and obtain the best decision, and then transmit it to the target farmers via the internet. The growth status of poultry is under a comprehensive action of elements such as farm environment and nutrition conditions. The poultry growth, environment control and diseases diagnosis are researched in a system. After operating and testing, the steadiness, effectiveness and reliability of the system is tested and verified.

\section{References}

[1] Chen Yifei. Exploration into the Relationship between Intelligent Control of Large Complex Agricultural System and Internet of Agriculture [J]. Agriculture Network Information, 2012(2): 8-12.

[2] Du Zhiguo, Hu Dahui. Poultry Diseases Diagnosis Expert System Based on Knowledge Base [J]. Journal of Agricultural Mechanization Research, 2009 (9): 165-167.

[3] Yipeng Sun, Yuanyuan Tan,Kai Wei, Honglei Sun,Yi Shi,Juan Pu, Hanchun Yang,George F. Gao,Yanbo Yin,Wenhai Feng,Daniel R. Perez, Jinhua Liua.Amino Acid 316 of Hemagglutinin and the Neuraminidase Stalk Length Influence Virulence of H9N2 Influenza Virus in Chickens and Mice, Journal of Virology, 2013, 87 (5) , 2963-2968.

[4] Qiao Jinyou. Design and Development of Agricultural Mechanized Production Expert System [D]. An Academic Dissertation of Northeast Agricultural University. 2007.

[5] Xu Xingang. Research Progress of Poultry Diseases Computer Diagnosis Expert System[J]. China Animal Health, 2004:49-50.

[6] Yin Y, Cortey M, Zhang Y, Cui S, Dolz R, Wang J, Gong Z. Molecular characterization of Newcastle disease viruses in Ostriches (Struthio camelus L.): Further evidences of recombination within avian paramyxovirus type 1. Veterinary Microbiology ,2011,03:324-329

[7] Jiangtao Xu, Zhenmei Zhang, Yanbo Yin, Shangjin Cui, Shouzhen Xua, Yanyan Guo, Jida Li, Jianlin Wang, Xingcai Liu, Limin Han..Development of reverse-transcription loop-mediated isothermal amplification for the detection of infectious bursal disease virus. J Virol Methods, 2009,12: 267-271

[8] Yang Jun, Qin Xiaojun, Wang Cheng. Design of Farm Environment Monitoring System Based on Expert System[J]. Journal of Agricultural Mechanization Research, 2007(6):163-169.

[9] Zhong Changle, Zhong Yong, Ma Chunquan, et al. Design and Implementation of Poultry Disease Diagnosis Expert System Based on Spring Framework [J]. Computer Application and Software, 2010,27(4):87-89. 